\title{
POTÁSSIO NO DESENVOLVIMENTO INICIAL DA SOQUEIRA DE CANA CRUA ${ }^{1}$
}

\author{
Rilner Alves Flores ${ }^{2}$, Renato de Mello Prado ${ }^{2}$, Lucas Sanches Politi² ${ }^{2}$ Thiago Batista Firmato de Almeida ${ }^{2}$
}

\begin{abstract}
POTASSIUM IN THE EARLY DEVELOPMENT

STAGE OF UNBURNED SUGARCANE RATOON

In the mechanized harvesting without the use of fire, there is deposition of straw on the soil surface, which may be recycled and reduce the potassium fertilization for sugarcane, if compared to the burned sugarcane, what may reflect in a lower production cost. This study aimed to evaluate the potassium application effect in the early development of first cutting sugarcane ratoon (SP 89-1115), in an unburned harvesting system. The experiment was installed in a first sugarcane ratoon area, in a clay Oxisol. The treatments consisted of five $\mathrm{K}_{2} \mathrm{O}$ doses $\left(0 \mathrm{~kg} \mathrm{ha}^{-1}\right.$, $32.5 \mathrm{~kg} \mathrm{ha}^{-1}, 65.0 \mathrm{~kg} \mathrm{ha}^{-1}, 130.0 \mathrm{~kg} \mathrm{ha}^{-1}$, and $\left.195.0 \mathrm{~kg} \mathrm{ha}^{-1}\right)$, as $\mathrm{KCl}$, applied in 2009, in a randomized blocks design with five replications. The growth variables measured were number of tillers, plant height, and stem diameter, 120 days after budding. The potassium application provided a linear increase in the soil potassium content, at the $0-0.20 \mathrm{~m}$ and $0.20-0.40 \mathrm{~m}$ depth layers, reaching, respectively, $0.18 \mathrm{cmol}_{\mathrm{c}} \mathrm{dm}^{-3}$ and $0.12 \mathrm{cmol}_{\mathrm{c}} \mathrm{dm}^{-3}$, for the highest $\mathrm{K}$ dose. The $\mathrm{K}$ fertilization did not affect the number of tillers and stem diameter, but it affected plant height, which reached its highest value at $195.0 \mathrm{~kg} \mathrm{ha}^{-1}$ of $\mathrm{K}_{2} \mathrm{O}$.
\end{abstract}

KEY-WORDS: Saccharum spp.; sugarcane biometrics; plant nutrition.

\section{INTRODUÇÃO}

Com a grande expansão do mercado sucro-alcooleiro e a incorporação de novas áreas, geralmente de baixa fertilidade, para a produção de açúcar e energia renovável, estudos sobre a cultura da cana-de-açúcar têm se intensificado, com o objetivo de aumentar a potencialidade das variedades, tornando-as produtivas, com novas técnicas de manejo e monitoramento das exigências nutricionais da planta, a fim de se recuperar e manter a fertilidade, para alcançar produções economicamente viáveis (Felipe 2008, Feltrin et al. 2010).

\section{RESUMO}

Na colheita mecanizada sem o uso de fogo ocorre deposição de palhada na superfície do solo, a qual pode ser reciclada e reduzir a adubação potássica para a cana-de-açúcar, em relação à cana queimada, o que pode refletir em menor custo de produção. Objetivou-se avaliar o efeito da aplicação de potássio no desenvolvimento inicial da soqueira de primeiro corte da cana-de-açúcar (variedade SP 89-1115), em sistema de colheita sem despalha a fogo. O experimento foi instalado em uma área de primeira soqueira de cana-de-açúcar, cultivada em Latossolo Vermelho, textura argilosa. Os tratamentos foram constituídos por cinco doses de $\mathrm{K}_{2} \mathrm{O}\left(0 \mathrm{~kg} \mathrm{ha}^{-1} ; 32,5 \mathrm{~kg} \mathrm{ha}^{-1} ; 65,0 \mathrm{~kg} \mathrm{ha}^{-1}\right.$; $130,0 \mathrm{~kg} \mathrm{ha}^{-1}$; e 195,0 $\mathrm{kg} \mathrm{ha}^{-1}$ ), na forma de KCl, aplicado em 2009 , dispostos em blocos casualizados e com cinco repetições. As variáveis de crescimento avaliadas foram: número de perfilhos, altura de planta e diâmetro de colmo, aos 120 dias após o brotamento. A aplicação de potássio proporcionou incremento com ajuste linear do teor de potássio no solo, nas camadas $0-0,20 \mathrm{me} 0,20-0,40 \mathrm{~m}$ de profundidade, atingindo $0,18 \mathrm{cmol} \mathrm{dm}_{c}^{-3}$ e $0,12 \mathrm{cmol}_{c} \mathrm{dm}^{-3}$, respectivamente, para a maior dose de $\mathrm{K}$. A aplicação de $\mathrm{K}$ não afetou o número de perfilhos e diâmetro de colmo, mas influenciou a altura, atingindo maior valor na dose de $195,0 \mathrm{~kg} \mathrm{ha}^{-1}$ de $\mathrm{K}_{2} \mathrm{O}$.

PALAVRAS-CHAVE: Saccharum spp.; biometria da cana-deaçúcar; nutrição de plantas.

A colheita de cana crua, sem despalha a fogo, vem crescendo de forma significativa no País, resultando, normalmente, no acúmulo de grandes quantidades de matéria seca (palhada) sobre a superfície do solo (Korndörfer \& Oliveira 2005). Em regiões canavieiras do Brasil e do mundo, onde a precipitação é pequena ou irregular, a presença da palhada sobre o solo também tem contribuído para elevar a produtividade da cana-de-açúcar (Ball-Coelho et al. 1993), principalmente por diminuir no solo a perda de água por evaporação e aumentar a infiltração da água.

No Estado de São Paulo, estima-se que a massa de palhada que permanece sobre o solo após o corte

1. Trabalho recebido em abr./2011 e aceito para publicação em mar./2012 (nº registro: PAT 13977).

2. Universidade Estadual Paulista "Júlio de Mesquita Filho", Faculdade de Ciências Agrárias e Veterinárias, Departamento de Solos e Adubos, Jaboticabal, SP, Brasil.E-mails: rilner1@hotmail.com,rmprado@fcav.unesp.br, nardosp@hotmail.com, thibalmeida@gmail.com. 
da cana apresente variação de 10-20 tha-1 de matéria seca, a qual possui uma alta relação C:N (Trivelin et al. 1995, Cantarella 1998), porém, com taxa de mineralização lenta (Oliveira et al. 1999). Entretanto, estes últimos autores, estudando a liberação do $\mathrm{K}$ presente na palhada, concluíram que o potássio foi o elemento mais liberado pela palhada de cana (média de $93 \%$ ), de um ano para o outro.

Sabe-se que os solos tropicais brasileiros apresentam baixo teor de potássio trocável (Ernani et al. 2007, Benites et al. 2010) e que a cana-de-açúcar responde de forma expressiva à aplicação do fertilizante potássico, nestas condições (Korndörfer \& Oliveira 2005).

O potássio é um nutriente importante para a cana-de-açúcar, sendo o mais extraído pela cultura (Malavolta 1994), principalmente pela cana-soca (Korndörfer \& Oliveira 2005), além de desempenhar várias funções, como regulação da turgidez do tecido, ativação enzimática, abertura e fechamento de estômatos, transporte de carboidratos, transpiração, resistência a geadas, seca, doenças e ao acamamento. A cana-de-açúcar apresenta, ainda, consumo de luxo, ou seja, mesmo absorvendo o $\mathrm{K}$ em altas quantidades, não há prejuízo à produtividade (Malavolta 1980).

Atualmente, é crescente a realização da colheita mecanizada da cana-de-açúcar, principalmente no Estado de São Paulo, em detrimento à colheita com despalha a fogo, implicando, assim, na necessidade de estudos mais detalhados a respeito da adubação potássica, mediante esta mudança no sistema de cultivo. Aliado a este fato, e apesar da importância do potássio para a cultura da cana-de-açúcar, poucos são os trabalhos na literatura sobre adubação com este nutriente na soqueira, com colheita sem a despalha a fogo, especialmente quando avaliado o desenvolvimento inicial da cultura. Diante disto, faz-se necessário realizar novos trabalhos sobre os efeitos do potássio na cana-de-açúcar, especialmente para as soqueiras cultivadas sem o uso da despalha a fogo.

Assim, este trabalho objetivou verificar o efeito da aplicação de doses crescentes de potássio no desenvolvimento inicial em soqueira de primeiro corte, em sistema de colheita sem despalha a fogo.

\section{MATERIAL E MÉTODOS}

O experimento foi desenvolvido em soqueira de cana-de-açúcar, variedade SP89-1115, localizada em Jaboticabal (SP), na fazenda Santo Antônio, em Latossolo Vermelho distrófico, textura argilosa (Embrapa 2006).

Para a caracterização da área e determinação das doses de fertilizantes, foram coletadas 15 subamostras de terra, para constituir a amostra composta, na camada $0-0,20 \mathrm{~m}$ de profundidade, para a análise química, visando à fertilidade, conforme métodos descritos por Raij et al. (2001), apresentando os seguintes resultados: $\mathrm{pH}: 5,0$; M.O.: $24 \mathrm{~g} \mathrm{dm}^{-3}$; P: $15 \mathrm{mg} \mathrm{dm}^{-3} ; \mathrm{K}: 0,07 \mathrm{cmol}_{\mathrm{c}} \mathrm{dm}^{-3}$; Ca: $0,30 \mathrm{cmol}_{\mathrm{c}} \mathrm{dm}^{-3}$; $\mathrm{Mg}: 1,6 \mathrm{cmol}_{\mathrm{c}} \mathrm{dm}^{-3} ; \mathrm{H}+\mathrm{Al}: 3,8 \mathrm{cmol}_{\mathrm{c}} \mathrm{dm}^{-3}$; SB: $4,67 \mathrm{cmol}_{\mathrm{c}} \mathrm{dm}^{-3}$; T: $8,47 \mathrm{cmol}_{\mathrm{c}} \mathrm{dm}^{-3}$; e V: $55 \%$.

O delineamento experimental adotado foi o de blocos casualizados, com cinco tratamentos e cinco repetições. Cada parcela foi composta por cinco linhas de 10,0 m de comprimento (espaçamento de $1,5 \mathrm{~m}$ entrelinhas), sendo as três linhas centrais consideradas úteis, no momento de cada amostragem.

Para o cultivo da soqueira de cana-de-açúcar, os tratamentos foram compostos por cinco doses de potássio, sendo utilizado, como referência, o teor de $\mathrm{K}^{+}$trocável no solo, com expectativa de produção variando entre $80 \mathrm{t} \mathrm{ha}^{-1}$ e $100 \mathrm{t} \mathrm{ha}^{-1}$, utilizando-se a dose de referência de $130 \mathrm{~kg} \mathrm{ha}^{-1}$ de $\mathrm{K}_{2} \mathrm{O}$, indicada para o Estado de São Paulo (Spironello et al. 1997), na forma de cloreto de potássio $\left(60 \%\right.$ de $\left.\mathrm{K}_{2} \mathrm{O}\right)$. Assim, as doses de $\mathrm{K}_{2} \mathrm{O}$ (tratamentos) foram as seguintes: $0 \mathrm{~kg} \mathrm{ha}^{-1} ; 32,5 \mathrm{~kg} \mathrm{ha}^{-1} ; 65,0 \mathrm{~kg} \mathrm{ha}^{-1} ; 130,0 \mathrm{~kg} \mathrm{ha}^{-1}$; e $195,0 \mathrm{~kg} \mathrm{ha}^{-1}$, correspondendo, respectivamente, a $0 \%, 25 \%, 50 \%, 100 \%$ e $150 \%$ da dose de referência (130 kg ha-1 de $\mathrm{K}_{2} \mathrm{O}$ ).

Foram aplicados, também, $30 \mathrm{~kg} \mathrm{ha}^{-1} \mathrm{de}_{2} \mathrm{O}_{5}$, na forma de super triplo, e $100 \mathrm{~kg} \mathrm{ha}^{-1} \mathrm{de} \mathrm{N}$, na forma de ureia (mesma dose para todos os tratamentos), ao lado da linha da soqueira da cana-de-açúcar, sem incorporação, conforme indicações de Spironello et al. (1997).

Foi realizada a amostragem da palhada presente no momento da instalação do experimento, a partir de três pontos aleatórios, com o auxílio de um quadro metálico de 1,0 $\mathrm{m}^{2}$ de área, obtendo-se 13,9 t ha ${ }^{-1} \mathrm{de}$ biomassa ( $16,5 \%$ de umidade), e realizou-se a análise química conforme metodologia descrita por Bataglia et al. (1983), determinando-se os teores de macro e micronutrientes $\left(\mathrm{N}=3,5 \mathrm{~g} \mathrm{~kg}^{-1} ; \mathrm{P}=0,5 \mathrm{~g} \mathrm{~kg}^{-1}\right.$; $\mathrm{K}=1,2 \mathrm{~g} \mathrm{~kg}^{-1} ; \mathrm{Ca}=3,1 \mathrm{~g} \mathrm{~kg}^{-1} ; \mathrm{Mg}=0,7 \mathrm{~g} \mathrm{~kg}^{-1}$; $\mathrm{S}=1,6 \mathrm{~g} \mathrm{~kg}^{-1} ; \mathrm{Cu}=3,0 \mathrm{mg} \mathrm{kg}^{-1} ; \mathrm{Fe}=346,0 \mathrm{mg} \mathrm{kg}^{-1}$; $\left.\mathrm{Mn}=85,0 \mathrm{mg} \mathrm{kg}^{-1} ; \mathrm{Zn}=5,0 \mathrm{mg} \mathrm{kg}^{-1} ; \mathrm{B}=7,9 \mathrm{mg} \mathrm{kg}^{-1}\right)$. A temperatura média e a precipitação ocorrida no período de condução do experimento foram $24^{\circ} \mathrm{C}$ e $1.450 \mathrm{~mm}$, respectivamente. 
Aos quatro meses após a brotação (06/03/2010), foram feitas as avaliações de crescimento e/ou desenvolvimento, sendo considerado o número de perfilhos em 1,0 m da linha da cultura, e, a partir de 10 plantas aleatórias nas linhas úteis de cada parcela, foi medida a altura das plantas (da base do colmo até a primeira bainha visível da planta) e o diâmetro dos colmos, com o auxílio de um paquímetro.

Aos seis meses após a aplicação dos tratamentos, foi realizada amostragem de solo, próxima à linha da cultura (faixa de adubação), nas camadas 0-0,20 m e 0,20-0,40 $\mathrm{m}$ de profundidade, a partir de 10 pontos aleatórios nas linhas úteis de cada parcela. Para as avaliações analíticas nas amostras de terra, para a determinação do potássio trocável no solo, foi utilizada metodologia descrita por Raij et al. (2001).

Os dados obtidos foram submetidos a análise de variância e, caso significativa $(p<0,05)$, realizou-se um estudo de regressão polinomial, com o auxílio do pacote estatístico AgroEstat (Barbosa \& Maldonado Júnior 2011).

\section{RESULTADOS E DISCUSSÃO}

Os teores de potássio no solo, nas camadas 0-0,20 $\mathrm{m}$ e 0,20-0,40 $\mathrm{m}$ de profundidade, foram afetados pela aplicação de doses crescentes de potássio, bem como o valor da saturação de bases (V\%), na camada 0-0,20 m (Tabela 1).

A aplicação de potássio na soqueira de cana-de-açúcar afetou a concentração de potássio do solo analisado aos seis meses após a aplicação dos tratamentos, na camada superficial e subsuperficial, observando-se que a aplicação de doses crescentes de potássio proporcionou incremento, com ajuste linear, do teor de potássio do solo das camadas 0-0,20 $\mathrm{m}$ e $0,20-0,40 \mathrm{~m}$ de profundidade, atingindo, na maior dose de potássio aplicada, $0,18 \mathrm{cmol}_{\mathrm{c}} \mathrm{dm}^{-3}$ e $0,12 \mathrm{cmol}_{\mathrm{c}} \mathrm{dm}^{-3}$, respectivamente (Figura 1). Este resultado foi semelhante aos obtidos por Silva (2010) e Rossetto et al. (2004), os quais constataram que a aplicação de potássio incrementou, com ajuste linear, o teor de potássio do solo.

O aumento da concentração do macronutriente na camada superficial do solo, em função da aplicação de doses crescentes de $\mathrm{K}_{2} \mathrm{O}$, já era esperado, uma vez que, em solos argilosos, com alta capacidade de troca de cátions (CTC), a perda de potássio por lixiviação é pequena (Mielniczuk 1982). Por outro lado, a concentração inicial de potássio no solo (antes da implantação do experimento) foi classificada como baixa (Raij \& Cantarella 1997), o que implicaria em maior possibilidade de resposta da cultura à aplicação do fertilizante potássico (Silva et al. 2007), pois,

Tabela 1. Análise do solo, nas camadas 0-0,20 m e 0,20-0,40 m de profundidade, em função da aplicação de doses crescentes de potássio, em soqueira avaliada aos 180 dias após o brotamento (Jaboticabal, SP, 2010).

\begin{tabular}{|c|c|c|c|c|c|c|c|c|c|c|}
\hline \multicolumn{11}{|c|}{ Profundidade de $0-0,20 \mathrm{~m}$} \\
\hline Dose de potássio & \multirow{2}{*}{$\mathrm{pH}$} & M.O. & $\mathrm{P}$ & $\mathrm{K}$ & $\mathrm{Ca}$ & $\mathrm{Mg}$ & $\mathrm{H}+\mathrm{Al}$ & SB & $\mathrm{T}$ & $\mathrm{V}$ \\
\hline $\mathrm{kg} \mathrm{ha}^{-1}$ de $\mathrm{K}_{2} \mathrm{O}$ & & $\mathrm{g} \mathrm{dm}^{-3}$ & $\mathrm{mg} \mathrm{dm} \mathrm{m}^{-3}$ & \multicolumn{6}{|c|}{$-\mathrm{cmol}_{\mathrm{c}} \mathrm{dm}^{-3}$} & $\%$ \\
\hline 0,0 & 5,1 & 31,0 & 8,4 & 0,07 & 3,20 & 1,36 & 4,42 & 4,6 & 9,0 & 51 \\
\hline 32,5 & 5,2 & 30,2 & 8,6 & 0,09 & 3,38 & 1,44 & 3,98 & 4,9 & 8,9 & 55 \\
\hline 65,0 & 5,2 & 29,6 & 9,6 & 0,10 & 3,58 & 1,58 & 3,82 & 5,2 & 9,1 & 58 \\
\hline 130,0 & 5,1 & 30,0 & 9,4 & 0,13 & 3,28 & 1,42 & 4,40 & 4,8 & 9,2 & 52 \\
\hline 195,0 & 5,1 & 30,6 & 9,0 & 0,18 & 3,36 & 1,54 & 4,40 & 5,0 & 9,5 & 53 \\
\hline Teste F & ns & ns & ns & $* *$ & ns & $*$ & ns & ns & ns & $*$ \\
\hline C.V. $(\%)$ & 2,9 & 4,4 & 10,4 & 16,2 & 8,4 & 7,4 & 10,1 & 6,4 & 5,5 & 5,6 \\
\hline \multicolumn{11}{|c|}{ Profundidade de $0,20-0,40 \mathrm{~m}$} \\
\hline Dose de potássio & \multirow{2}{*}{$\mathrm{pH}$} & M.O. & $\mathrm{P}$ & $\mathrm{K}$ & $\mathrm{Ca}$ & $\mathrm{Mg}$ & $\mathrm{H}+\mathrm{Al}$ & SB & $\mathrm{T}$ & $\mathrm{V}$ \\
\hline $\mathrm{kg} \mathrm{ha}^{-1}$ de $\mathrm{K}_{2} \mathrm{O}$ & & $\mathrm{g} \mathrm{dm}^{-3}$ & $\mathrm{mg} \mathrm{dm}^{-3}$ & \multicolumn{6}{|c|}{$-\mathrm{cmol}_{\mathrm{c}} \mathrm{dm}^{-3}$} & $\%$ \\
\hline 0,0 & 4,7 & 20,8 & 15,2 & 0,07 & 2,08 & 1,06 & 4,56 & 3,2 & 7,7 & 41 \\
\hline 32,5 & 4,8 & 22,4 & 15,6 & 0,09 & 2,04 & 1,07 & 4,40 & 3,2 & 7,5 & 42 \\
\hline 65,0 & 4,8 & 21,2 & 17,8 & 0,10 & 1,88 & 1,14 & 4,30 & 3,1 & 7,4 & 42 \\
\hline 130,0 & 4,7 & 20,4 & 18,0 & 0,10 & 1,84 & 1,15 & 4,60 & 3,1 & 7,7 & 40 \\
\hline 195,0 & 4,8 & 22,2 & 18,8 & 0,12 & 2,12 & 1,16 & 4,50 & 3,4 & 7,9 & 43 \\
\hline Teste $\mathrm{F}$ & ns & ns & $* *$ & $* *$ & ns & $\mathrm{ns}$ & ns & ns & ns & $\mathrm{ns}$ \\
\hline C.V. $(\%)$ & 2,8 & 7,8 & 6,0 & 12,1 & 9,1 & 7,7 & 6,0 & 7,6 & 4,9 & 5,5 \\
\hline
\end{tabular}

M.O.: Matéria orgânica. SB: Soma de bases. *, ** e ns: significativo a 5\% e 1\% e não significativo, respectivamente, pelo teste F. 
(a)

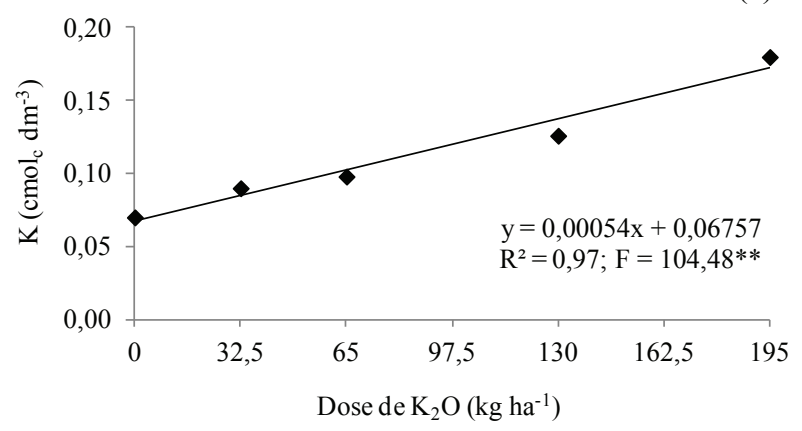

(b)

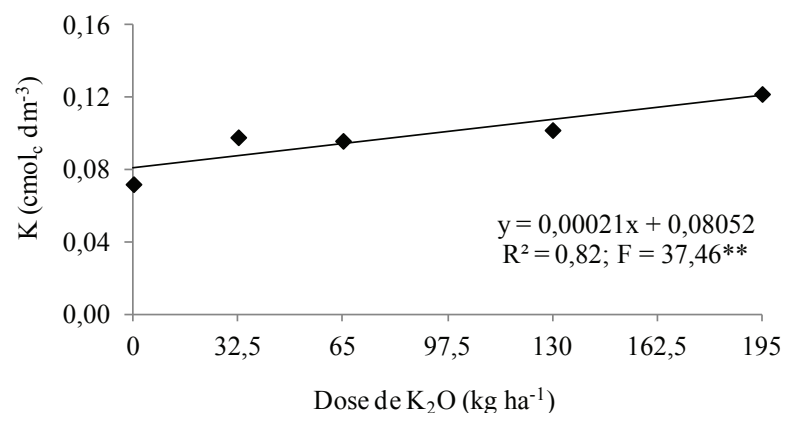

Figura 1. Teor de K no solo, na camada 0-20 cm (a) e 20-40 cm (b) de profundidade, em função de doses crescentes de potássio, em soqueira de cana-de-açúcar (Jaboticabal, SP, 2010).

segundo Silva et al. (2005), em solos que apresentam teor deste nutriente superior a $150 \mathrm{mg} \mathrm{dm}^{-3}$, não se recomenda a aplicação do fertilizante potássico. Porém, a influência da precipitação na região, ocorrida no intervalo entre a aplicação dos tratamentos e o momento da avaliação do potássio trocável no solo, pode ter possibilitado a solubilização do potássio aplicado e o possível aprofundamento do íon no perfil do solo das maiores doses, reduzindo os teores deste nutriente no solo.

A saturação de bases (V\%) apresentou efeito quadrático, atingindo $56 \%$, na dose de $110 \mathrm{~kg} \mathrm{ha}^{-1} \mathrm{de}$ $\mathrm{K}_{2} \mathrm{O}$, com valor do teste $\mathrm{F}$ igual a 4,55, porém, com baixo valor de $\mathrm{R}^{2}$ (0,30 de ajuste).

Houve incremento na altura das plantas, em função das doses de potássio aplicadas (Tabela 2). Verificou-se incremento com ajuste linear na altura das plantas, em função das doses de K, atingindo-se

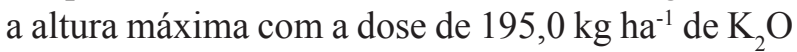
(Figura 2).

Tabela 2. Altura, número e diâmetro de perfilho da cana-deaçúcar, em função da aplicação de doses crescentes de potássio, em soqueira avaliada aos 120 dias após o brotamento (Jaboticabal, SP, 2010).

\begin{tabular}{|c|c|c|c|}
\hline $\begin{array}{c}\text { Doses de } \\
\mathrm{K}_{2} \mathrm{O}\end{array}$ & Altura & \multirow{2}{*}{$\begin{array}{l}\text { Número de } \\
\text { perfilhos }\end{array}$} & $\begin{array}{l}\text { Diâmetro do } \\
\text { perfilho }\end{array}$ \\
\hline $\mathrm{kg} \mathrm{ha}^{-1}$ & $\mathrm{~cm}$ & & $\mathrm{~mm}$ \\
\hline 0,0 & 273 & 29,0 & 28,7 \\
\hline 32,5 & 277 & 27,8 & 28,4 \\
\hline 65,0 & 272 & 29,4 & 28,6 \\
\hline 130,0 & 278 & 29,2 & 29,2 \\
\hline 195,0 & 279 & 30,8 & 29,4 \\
\hline Teste F & $5,52 * *$ & $0,37^{\mathrm{ns}}$ & $0,59^{\text {ns }}$ \\
\hline C.V. $(\%)$ & 1,1 & 13,4 & 4,2 \\
\hline
\end{tabular}

El-Tilib et al. (2004) também observaram efeito significativo para a altura das plantas na soqueira de cana-de-açúcar, em função da aplicação de potássio em todas as fases de crescimento da cultura avaliadas (4 até 10 meses após o brotamento da soqueira), sendo a melhor resposta obtida com a dose de $86 \mathrm{~kg} \mathrm{ha}^{-1}$ de $\mathrm{K}_{2} \mathrm{O}$. Os mesmos autores concluíram que o melhor equilíbrio dos nutrientes no solo, atingido nesta dose, proporcionou maiores incrementos na altura das soqueiras da cana-de-açúcar.

A aplicação de potássio não afetou o número de perfilhos e o diâmetro de colmos, na soqueira de cana-de-açúcar (Tabela 2). A ausência do efeito do potássio nas variáveis de crescimento da cultura provavelmente ocorreu devido à baixa exigência nutricional da cultura, nesta fase, pois, segundo Coelho \& Verlengia (1973), trata-se de uma fase de crescimento lento. Neste sentido, na literatura, o efeito do potássio, nas variáveis de crescimento como diâmetro de caule e número de colmos da cana-de-

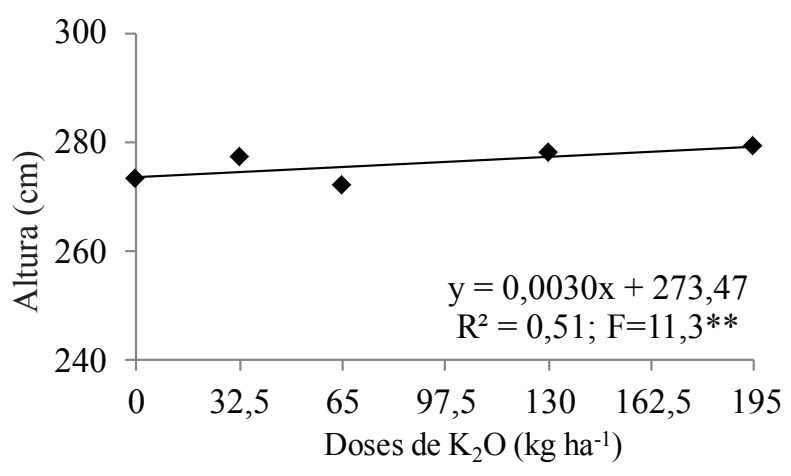

Figura 2. Altura da planta, em função de doses crescentes de potássio, em soqueira de cana-de-açúcar (Jaboticabal, SP, 2010). 
-açúcar, apenas foi verificado tardiamente, aos dez meses após a brotação da planta (Uchôa et al. 2009).

Em estudo semelhante, Silva (2010) também observou que a aplicação de doses crescentes de potássio não afetou o número de perfilhos, aos quatro meses após a brotação. No entanto, na avaliação feita no oitavo mês após a brotação, não foi observado efeito significativo para altura, diâmetro de colmo e número de perfilhos, em função da aplicação de doses crescentes de potássio. Além disto, Teixeira (2005) e Felipe (2008) não observaram incrementos significativos para o número de colmos e para o diâmetro do colmo, respectivamente.

Contrariamente ao presente estudo, Dantas Neto et al. (2006) e Shukla et al. (2009), em estudos em soqueiras de cana-de-açúcar, observaram aumento no número de colmos, em função do aumento de doses de potássio aplicadas, e Silva (2010) não observou efeito significativo para a altura, em função das doses crescentes de potássio aplicadas, aos 12 meses após o brotamento da primeira soqueira da cana-de-açúcar. Já Orlando Filho et al. (1993) constataram que a deficiência de potássio não alterou o perfilhamento, porém, provocou diminuição no diâmetro médio e altura dos colmos.

Em geral, o aumento de produtividade deve-se à integração entre a altura, diâmetro e número de colmos, e pequenos acréscimos em cada uma destas categorias nem sempre são diferenciados pela análise estatística.

A ausência de efeito da aplicação de doses de potássio no número de perfilhos e no diâmetro da cana-soca, durante o crescimento inicial da cultura, possivelmente deveu-se ao pouco tempo de experimentação, que pode ter sido insuficiente para demonstrar o real potencial do potássio na melhoria do desenvolvimento da cultura da cana-de-açúcar. Segundo Coelho \& Verlengia (1973), a exigência nutricional da cultura, no início do crescimento, é relativamente baixa, aumentando, intensamente, entre 150 e 360 dias após a brotação.

Nota-se a existência de poucos trabalhos relacionados ao crescimento de soqueiras de cana-de-açúcar com sistema de colheita sem despalha a fogo, e que estes trabalhos restringem as análises de crescimento realizadas ao momento da colheita.

\section{CONCLUSÕES}

1. A aplicação de potássio incrementou o teor de potássio no solo das camadas $0-0,20 \mathrm{~m}$ e
0,20-0,40 $\mathrm{m}$ de profundidade, atingindo, na maior dose de potássio aplicada, $0,173 \mathrm{cmol}_{\mathrm{c}} \mathrm{dm}^{-3} \mathrm{e}$ $0,121 \mathrm{cmol}_{\mathrm{c}} \mathrm{dm}^{-3}$, respectivamente.

2. A aplicação de potássio não alterou o número de perfilhos e diâmetro de colmos da soqueira de cana-de-açúcar, entretanto, aumentou a altura, a qual atingiu maior valor com $195,0 \mathrm{~kg} \mathrm{ha}^{-1} \mathrm{de}_{2} \mathrm{O}$.

\section{REFERÊNCIAS}

BALL-COELHO, B. et al. Residue management effects on sugarcane yield and soil properties in Northeastern Brazil. Agronomy Journal, Madison, v. 85, n. 5, p. 10041008, 1993.

BARBOSA, J. C.; MALDONADO JÚNIOR, W. AgroEstat: sistema para análise estatística de ensaios agronômicos. Versão 1.0. Jaboticabal: FCAV/Unesp, 2011.

BATAGLIA, O. C. et al. Métodos de análise química de plantas. Campinas: IAC, 1983. (Boletim técnico, 78).

BENITES, V. M. et al. Potássio, cálcio e magnésio. In: PROCHNOW, L. I.; CASARIN, V.; STIPP, S. R. (Eds.) Boas práticas para o uso eficiente de fertilizantes: nutrientes. Piracicaba: IPNI, 2010. p. 137-191.

CANTARELLA, H. Aplicação de nitrogênio em sistema de cana-crua. STAB - Açúcar, Álcool e Subprodutos, Piracicaba, v. 16, n. 1, p. 21-22, 1998.

COELHO, F. S.; VERLENGIA, F. Fertilidade do solo. 2. ed. Campinas: Instituto Campineiro de Ensino Agrícola, 1973.

DANTAS NETO, J. et al. Resposta da cana-de-açúcar, primeira soca, a níveis de irrigação e adubação de cobertura. Revista Brasileira de Engenharia Agrícola e Ambiental, Campina Grande, v. 10, n. 2, p. 283-288, 2006.

EL-TILIB, M. A.; ELNASIKH, M. H.; ELAMIN, E. A. Phosphorus and potassium fertilization effects on growth attributes and yield of two sugarcane varieties grown on three soil series. Journal of Plant Nutrition, New York, v. 27 , n. 4, p. 663-699, 2004.

EMPRESA BRASILEIRA DE PESQUISA AGROPECUÁRIA (Embrapa). Centro Nacional de Pesquisa de Solos. Sistema brasileiro de classificação de solos. Rio de Janeiro: Embrapa, 2006.

ERNANI, P. R.; ALMEIDA, J. A.; SANTOS, F. C. Potássio. In: NOVAIS, R. F. et al. (Eds.). Fertilidade do Solo. Viçosa: Sociedade Brasileira de Ciência do Solo, 2007. p. 551-594.

FELIPE, D. C. Produtividade da cana-de-açúcar (Saccharum oficinarum L.) submetida a diferentes épocas de plantio e a adubação mineral. 2008. 70 f. Dissertação 
(Mestrado em Agronomia)-Universidade Federal da Paraíba, Areia, 2008.

FELTRIN, M. S. et al. Adubação potássica na produtividade da soqueira de cana-de-açúcar colhida sem queima. Núcleus, Ituverava, v. 7, n. 1, p. 307-314, 2010.

KORNDÖRFER, G. H.; OLIVEIRA, L. A. O potássio na cultura da cana-de-açúcar. In: YAMADA, T.; ROBERTS, T. L. (Eds.). Potássio na agricultura brasileira. Piracicaba: ESALQ/USP, 2005. p. 469-490.

MALAVOLTA, E. Elementos de nutrição mineral de plantas. Piracicaba: Ceres, 1980.

MALAVOLTA, E. Importância da adubação na qualidade dos produtos: função dos nutrientes na planta. In: SÁ, M. E.; BUZZETI, S. Importância da adubação na qualidade dos produtos agrícolas. São Paulo: Ícone, 1994. p. 19-44.

MIELNICZUK, J. Avaliação da resposta das culturas ao potássio em ensaios de longa duração: experiências brasileiras. In: YAMADA, T.; MUZZILLI, O.; USHERWOOD, N. R. (Eds.). Potássio na agricultura brasileira. Piracicaba: Instituto da Potassa e Fosfato, 1982. p. 289-303.

OLIVEIRA, M. W. et al. Degradação da palha de canade-açúcar. Scientia Agricola, Piracicaba, v. 56, n. 4, p. 803-809, 1999.

ORLANDO FILHO, J. et al. Fontes de potássio na adubação da cana-de-açúcar: $\mathrm{KCl} \mathrm{e}_{2} \mathrm{SO}_{4}$. STAB: Açúcar, Álcool e Subprodutos, Piracicaba, v. 11, n. 6, p. 39-43, 1993.

RAIJ, B. V. et al. (Eds.). Análise química para avaliação da fertilidade do solo. Campinas: IAC, 2001.

RAIJ, B. V.; CANTARELLA, H. Outras culturas industriais. In: RAIJ, B. V. et al. (Eds.). Recomendações de adubação e calagem para o Estado de São Paulo. Campinas: IAC, 1997. p. 233-244.

ROSSETTO, R. et al. Calagem para cana-de-açúcar e sua interação com doses de potássio. Bragantia, Campinas, v. 63, n. 1, p. 105-119, 2004.
SHUKLA, S. K. et al. Potassium nutrition for improving stubble bud sprouting, dry matter partitioning, nutrient uptake and winter initiated sugarcane (Saccharum spp. hybrid complex) ratoon yield. European Journal of Agronomy, Oxford, v. 30, n. 1, p. 27-33, 2009.

SILVA, F. C. et al. Avaliação da adubação com nitrogênio e potássio em soqueiras de cana-de-açúcar sem queima. Campinas: Embrapa, 2007. (Boletim de pesquisa e desenvolvimento, 16).

SILVA, F. C. et al. Avaliação da adubação nitrogenada e potássica em cana-de-açúcar baseada em modelos. In: SIMPÓSIO SOBRE POTÁSSIO NA AGRICULTURA, 2., 2005, Piracicaba. Anais... Piracicaba: Potafos, 2005. p. $763-822$.

SILVA, T. M. R. Nutrição potássica na primeira soqueira de cana-de-açúcar cultivada em sistema de colheita sem despalha a fogo. 2010. 58 f. TCC (Graduação em Agronomia)-Faculdade de Ciências Agrárias e Veterinárias, Universidade Estadual Paulista, Jaboticabal, 2010.

SPIRONELLO, A. et al. Cana-de-açúcar. In: RAIJ, B. V. et al. (Eds.). Recomendações de adubação e calagem para o Estado de São Paulo. Campinas: Fundação IAC, 1997. p. 237-239. (Boletim técnico, 100).

TEIXEIRA, C. D. Adubação nitrogenada e potássica em cana-soca, em dois solos do Estado do Paraná. 2005. 56 f. Dissertação (Mestrado em Agronomia)-Universidade Federal do Paraná, Curitiba, 2005.

TRIVELIN, P. C. O.; VICTORIA, R. L.; RODRIGUES, J. C. Aproveitamento por soqueira de cana-de-açúcar de final de safra do nitrogênio da aquamônia- ${ }^{15} \mathrm{~N}$ e aplicado ao solo em complemento à vinhaça. Pesquisa Agropecuária Brasileira, Brasília, DF, v. 30, n. 12, p. 1375-1385, 1995.

UCHÔA, S. C. P. et al. Resposta de seis variedades de cana-de-açúcar a doses de potássio em ecossistema de Cerrado em Roraima. Ciências Agronômicas, Fortaleza, v. 40, n. 4, p. 505-513, 2009. 